2.

\title{
Berichtigung einiger Momente und Anachronismen in Sachen des „Resorcins".
}

\author{
Von Dr. Justus Andeer in Strassburg.
}

In Schmidt's Jahrbüchern der Gesammt-Medicin, Band 189 Seite 95 Jahrgang 1881 hat Herr Kobert in seiner Recension der Resorcin-Literatur rücksichtlich methodischer Prüfung des Resorcins Herrn Brieger die Priorltät vindicirt.

Auch in der „Berliner klinischen Wochensebrift" No. 5 Seite 69 und No. 14 Seite 202 Jahrgang 1881 finden sich Beferate, in welchen darauf hingewiesen wird, dass Herr Brieger zuerst die physiologische Wirksamkeit der Dihydroxylbenzole auseinandergesetzt und methodisch geprüft habe.

Ich möchte diese Angaben auf das Nachdrücklichste dabin berichtigen, dass ich nach Kenntnissnakme und Untersuchung aller drei Dihydroxylbenzole, dic ich als unbrauchbare "Antipyretica " schon 1877 hinreichend kannte, in eben demselben Jahre die Aufmerksamkeit des ärztlichen Publikums auf das "Resorcinum antisepticum, causticum et haemostaticum " als auf das entschieden bedeutsamste unter diesen Isomeren gelenkt habe Ueber das Brenzeatechin und Hydrochinon habe ich allerdings nfchts veröffentlicht. Die Angaben des Herrn Brieger, sowie mehrerer Berichterstatter haben demnach wohl für das Brenzcatechin und Hydrochinon Gültigkeit, durchaus aber nicht für das Resorcin. Für letzteres nehme ich auf Grond der Jahresberichte der schweizerischen naturforschenden Gesellschaft vom Jahre 1877 S. 148 und S. 323 für mich die Priorität in Anspruch, waren doch zur Ermittelung und Feststellung der pbarmakologischen Eigenschaften anticipirende physiologische Untersuchungen des Mittels nothwendig. Dass letztere so methodisch wie die von Herrn Brieger erst 1879 veröffentlichten waren, hat Herr Brieger selbst in einer Anmerkung des „Centralblattes für medicinische Wissenschaften" No. 37 Jahrgang 1880 zugegeben. Herr Brieger sagt dort:

"Anf die Angaben des Herrn Andeer näher einzutreten, liegt hier keine Veranlassung vor. Anstatt anzuführen, dass ich dieselben Versuche wie er angestellt und veröffentlicht habe, Versuche, die in ihrer Anordnung vielfach mit den seinigen übereinstimmen und deren Ergebnisse im Wesentlichen die gleichen sind, wie er sie erhalten hat, weiss er nur zwei ganz nebensăchliche Angaben zu berichtigen (?), *

Warum Herr Brieger das sehr viel und doch wieder nichtssagende Fragezeichen gesetzt hat, statt neue Versucbsreihen anzustellen und nach deren Ergebvissen entweder positiv auszusprechen, dass meine Versuche fehlerhafte seien, oder aber zuzugeben, dass in dem betreffenden Pankte seine Beobachtungen nicht zutreffend waren, ist unerfindlich! 